\title{
Five Really Easy Steps to Build a Homemade Low-Cost Simulator
}

\author{
João Moreira-Pinto, MD ${ }^{1,2}$, João Guilherme Silva, $M D^{3}$, \\ João Luís Ribeiro de Castro, $M D^{2}$, and Jorge Correia-Pinto, MD, PhD ${ }^{1,4}$
}

\begin{abstract}
Aim. The aim of this study was to evaluate how simple it is to build a homemade low-cost simulator using a simple 5-step scheme. Methods. A scheme explaining how to build an endoscopic surgery simulator in 5 easy steps was presented to 26 surgeons. The simulator required a pair of scissors and easy-to-find materials. Its total cost was less than $€ 35$. The participants assessed the simulator using common endoscopic training toys or ex vivo tissue and completed an anonymous query comparing it with other commercial simulators that they had experienced before. Results. In all, $\mathbf{8 4 . 6 \%}$ found the simulator really easy to build. Every participant felt that he or she could do the same simulator themselves. Comparing with other commercial available box simulators, the majority of participants found the homemade simulator easier to (a) mount and dismount, (b) transport, (c) clean, and (d) use when practicing alone. Conclusions. Anyone can build its own simulator for a small amount of money.
\end{abstract}

\section{Keywords}

ergonomics and/or human factors study; simulation; surgical education

\section{Introduction}

Surgery has undergone a big transformation in the past 3 decades. Since 1987, when Dr Philippe Mouret performed the first laparoscopic cholecystectomy, minimally invasive surgery started spreading worldwide. ${ }^{1}$ At the same time, surgeons were forced to acquire new skills in order to perform surgical procedures through endoscopic, indirect visualization.

Just as laboratory training and operative experience with open surgical technique allow surgeons to develop open surgical skills, laparoscopic skill acquisition requires practice. Gaining skills in laparoscopic surgery requires specific training. Besides animal hands-on courses, surgeons have to spend a great amount of time practicing in simulators. ${ }^{2}$ Indeed, dedicated conscientious laparoscopic skill practice has been shown to improve the clinical performance of even experienced laparoscopists. ${ }^{3}$

Commercially available endoscopic simulators are usually expensive and not available to the surgeon on a daily basis. For that, several homemade box simulators have been previously reported. ${ }^{4-8}$ However, a simple instruction guide to how to build one is lacking. For that, we designed a 5-step scheme showing how simple it is to build a homemade low-cost simulator.

\section{Material and Methods}

A 5-step scheme explaining how to build an endoscopic surgery simulator was presented to 26 surgeons ( 8 residents and 18 senior surgeons) from different specialties (pediatric surgery, general surgery, gynecology, and urology).

The endoscopic simulator required a laptop or desktop computer with 1 free USB port, a pair of scissors, and other easy-to-find cheap materials: a translucent plastic box, a webcam, a USB cable, adhesive tape, Velcro tape, and a rubber sheet (Figure 1).

We used a medium-size translucent plastic box $(25 \times$ $30 \times 40 \mathrm{~cm}$ ). Using the pair of scissors, we cut the center

\footnotetext{
'Life and Health Sciences Research Institute (ICVS), School of Health Sciences, University of Minho, Braga, Portugal

${ }^{2}$ Pediatric Surgery Department, Centro Hospitalar do Porto, Porto, Portugal

${ }^{3}$ General Surgery Department, Hospital Santa Maria Maior, Barcelos, Portugal

${ }^{4}$ Pediatric Surgery Department, Hospital de Braga, Braga, Portugal

\section{Corresponding Author:}

João Moreira-Pinto, Surgical Sciences Research Domain, Life and Health Sciences Research Institute (ICVS), School of Health Sciences, University of Minho-Campus de Gualtar, Braga 47 I0-057, Portugal Email: moreirapinto@ecsaude.uminho.pt
} 


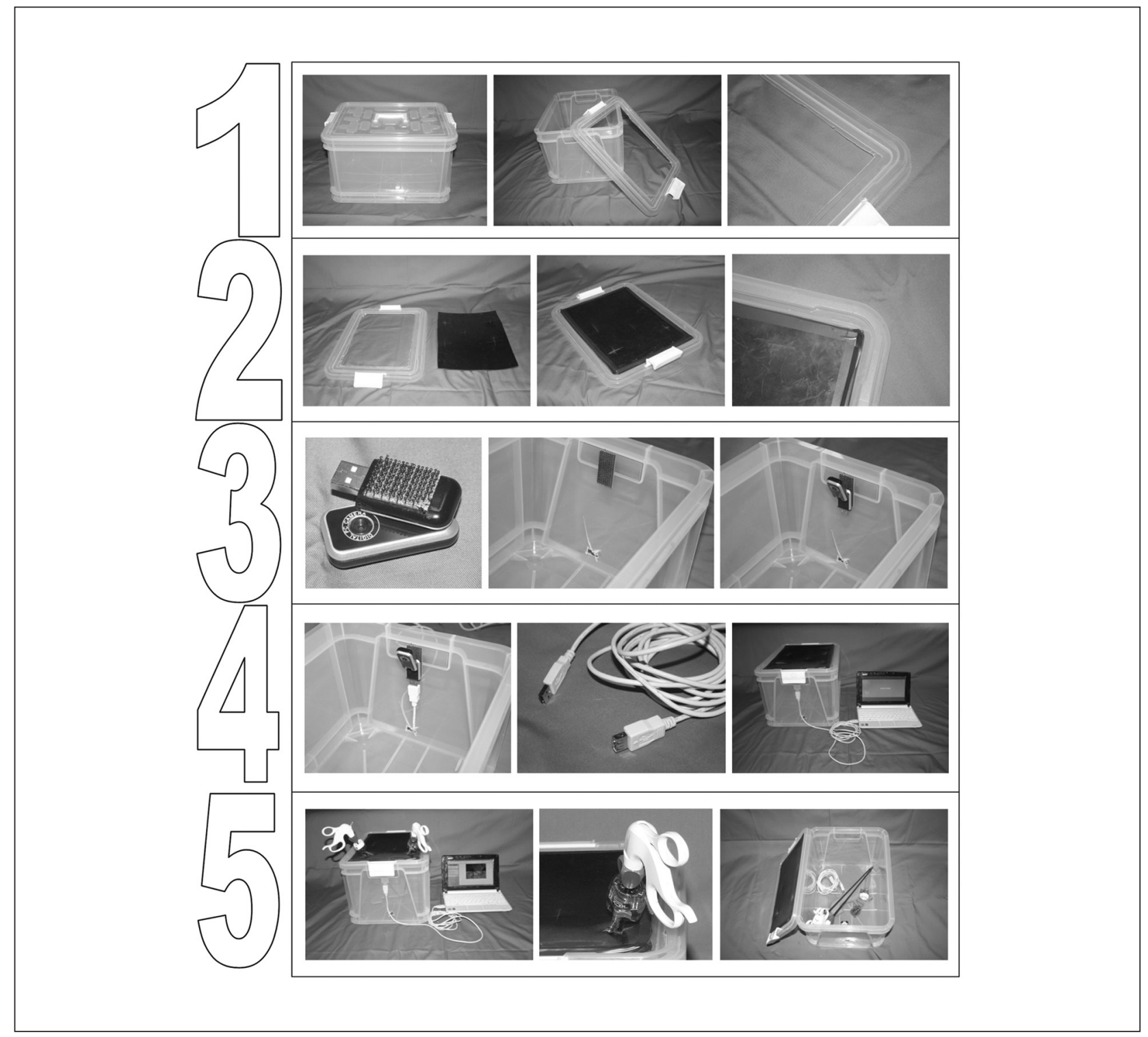

Figure I. How to build a homemade endoscopic simulator in 5 steps

(I) Cut the top of a medium-size translucent plastic box, leaving a $2 \mathrm{~cm}$ margin. (2) Cut a rubber sheet to fit the box's top, and tape them together. (3) Fix an articulated webcam to the box's inner anterior wall using Velcro tape. (4) Connect the webcam to your computer using a USB cable passed through a small hole in the plastic bottom, and point it to the centre of the box. (5) Close the box and insert 2 trocars in the rubber sheet and start practicing.

of the box's top leaving a $2 \mathrm{~cm}$ margin. Then, we cut the rubber sheet to fit the box's top, and we taped it together using strong adhesive tape. An articulated webcam (C-123B, Mitsai, Matosinhos, Portugal) was fixed to the inner anterior wall of the box using Velcro tape. The webcam was connected to the computer using an USB cable passed through a small hole in the bottom of the plastic box. We used a laptop computer running Windows XP operating system, which recognized the webcam automatically. By double clicking the webcam's icon on "My Computer" folder, one could get the preview image coming from the webcam. The articulated webcam optic was directed to the middle of the box, and various endoscopic toys and ex vivo animal tissues were put in the center of the image. After closing the box, two $5 \mathrm{~mm}$ trocars (Ethicon Endosurgery, Cincinnati, OH) were introduced through different incisions on the rubber sheet, depending on the surgeon's preference. Regular laparoscopic disposable instruments and trocars were used to perform different training tasks.

The total cost of this homemade endoscopic simulator was $€ 33.67$ (Table 1).

After looking at the 5-step scheme, the participants assessed the homemade endoscopic simulator using 
Table I. List of Materials and Its Prices

\begin{tabular}{lc}
\hline Materials & Price () \\
\hline Translucent plastic box $(25 \times 30 \times 40 \mathrm{~cm})$ & 4.45 \\
Rubber sheet $(21 \times 36 \mathrm{~cm})$ & 1.5 \\
Adhesive tape & 3.29 \\
Velcro tape & 4.95 \\
Webcam $(650 \times 480$ pixels) & 11.99 \\
USB (universal serial bus) cable & 7.49 \\
Total cost & 33.67 \\
\hline
\end{tabular}

common endoscopic training toys or ex vivo tissue. Then, they completed an anonymous query evaluating the 5 -step scheme and comparing the homemade endoscopic simulator to other commercially available simulators that they had experienced before, either during laparoscopy courses or in their hospital's training laboratory (Table 2).

\section{Results}

In all, $84.6 \%$ of the surgeons questioned found the simulator very easy to build, and the remaining $15.4 \%$ found it easy to build. All participants felt that they could build the same simulator on their own. All participants admitted that owning an endoscopic simulator is important for initial laparoscopy training. In fact, $69.2 \%$ of them answered that it was very important. Ninety-six percent agreed that the simulator was cheap, $4 \%$ found it was more or less expensive, and none found it expensive.

Participants' answers comparing the homemade endoscopic simulator to other commercially available box simulators are resumed in Table 3 . The majority of participants $(88.5 \%)$ found the homemade endoscopic simulator easier to mount and dismount, easier to transport $(88.4 \%)$, easier to clean $(80.4 \%)$, and easier to use $(84 \%)$ when practicing alone. The majority of the participants found quality of light, image, material resistance, and overall endoscopic environment at least as good as the commercially available box simulators that they had experienced before.

\section{Discussion}

Various endoscopic simulators have been presented to improve laparoscopic surgeons' skill. Some of these simulators incorporate virtual reality software that both simulates an operative endoscopic environment and monitors surgeons' skills. ${ }^{9}$ The limitations of such complex devices are cost, mobility, and availability. Commercially available endoscopic simulators are very expensive. Not every hospital can afford one, and when there is that possibility, endoscopic simulators are usually stationed in an endoscopic training facility, where access is limited by time, scheduling, and availability of accessory equipment, such as video monitor, endoscopic camera, and laparoscopic instruments. ${ }^{7}$ For that, several homemade box simulators have been presented before. ${ }^{4-6}$ Recently, Chung et $\mathrm{al}^{7}$ reported the effectiveness of a cardboard box simulator with a webcam system for laparoscopy training. Beatty ${ }^{8}$ presented a similar simulator, but he used a translucent plastic box, making it more resistant and avoiding the need of a lamp inside the box. Having these in mind, we designed 5 steps to building a homemade endoscopic simulator, so that anyone could build one as well as mount and dismount it easily.

We designed a homemade endoscopic simulator with cheap and easy-to-find materials. A plastic box was preferred over other materials (eg, cardboard) because of its lightness, resistance, and easy washability. The former is especially important if one wants to train using ex vivo tissue. The box was translucent in order to receive light from the exterior, which proved to be enough for good visualization. There are webcams that have a light source incorporated that could obviate the need of translucent plastic, but these are harder to find for most surgeons. By fixing the webcam with Velcro tape, we made the simulator easy to mount/dismount, so that one could wash it after using ex vivo tissues. Finally, the rubber sheet over the top of the box mimics the thoracic/abdominal wall not only for its texture and bulging movements but also by blocking surgeon's direct visualization from the outside. The rubber sheet also permits inserting trocars in different places, depending on the tasks one intends to practice.

As pointed out by Chung et al, ${ }^{7}$ webcam permits zooming in, recording training sessions, and transmitting them through the Internet in real time, which opens new perspectives on laparoscopic training, such as telementoring, group discussions, and creation of hands-on courses at low cost.

Our aim was not only to make an easy-to-build laparoscopic simulator but also to design a 5-step scheme that would be easy for any surgeon to follow. By answering the query, all surgeons said that they could follow our scheme and make their own homemade simulator, and most of them said it was very easy to do. Of course, it would be better to actually see if they could do it by giving them the tools and materials that they would need but that would take us lot of plastic boxes, and probably surgeons would not be keen on spending time cutting the plastic box as we did while doing this study. From the answers we got, we found that most surgeons think that it is important to own an endoscopic simulator in order to develop initial laparoscopic skills.

We did not query participants which commercially available endoscopic box simulator they had experienced before for comparison, because most had trained in more than one and would not remember their names. So participants judged our homemade endoscopic simulator based on their 
Table 2. Query on Homemade Endoscopic Simulator

I. After looking at this 5-step scheme, how easy do you find it to reproduce?

(a) very easy, (b) easy, (c) medium difficulty, (d) difficult, (e) very difficult

2. After looking at this 5 -step scheme, could you make an endoscopic simulator of your own?

(a) yes, (b) no

3. How important is it to have your own endoscopic simulator for initial laparoscopic training?

(a) not important at all, (b) not important, (c) important, (d) very important

4. Do you find this homemade endoscopic simulator expensive?

(a) yes, (b) more or less, (c) no

5. Comparing to other commercially available endoscopic simulators, how do you find this homemade endoscopic simulator concerning

mounting/dismounting: (a) far worse, (b) worst, (c) same; (d) better, (e) much better

transporting: (a) much worse, (b) worst, (c) same, (d) better, (e) much better

cleaning: (a) much worse, (b) worst, (c) same, (d) better, (e) much better

resistance: (a) much worse, (b) worst, (c) same, (d) better, (e) much better

one surgeon practice: (a) much worse, (b) worst, (c) same, (d) better, (e) much better

image light: (a) much worse, (b) worst, (c) same, (d) better, (e) much better

overall endoscopic environment: (a) much worse, (b) worst, (c) same, (d) better, (e) much better

Table 3. Answers Comparing the Homemade Endoscopic Simulator to Commercially Available Ones ${ }^{\mathrm{a}}$

\begin{tabular}{|c|c|c|c|c|c|}
\hline & Much Worse & Worse & Same & Better & Much Better \\
\hline Mounting/dismounting & - & - & $3(1 \mathrm{I} .5)$ & $15(57.7)$ & $7(30.8)$ \\
\hline Transporting & - & - & $3(1 \mathrm{I} .5)$ & $16(61.5)$ & $7(26.9)$ \\
\hline Cleaning & - & - & $5(19.2)$ & $17(65.4)$ & $4(15.4)$ \\
\hline Resistance $^{\mathrm{b}}$ & - & $6(24.0)$ & $15(60.0)$ & $4(15.4)$ & - \\
\hline One-surgeon practice $^{a}$ & - & - & $4(16.0)$ & $14(56.0)$ & $7(28.0)$ \\
\hline Image light ${ }^{a}$ & - & $4(16.0)$ & $17(68.0)$ & $3(12.0)$ & I $(4.0)$ \\
\hline Endoscopic environment & $2(7.7)$ & $5(19.2)$ & $16(61.5)$ & $3(11.5)$ & - \\
\hline
\end{tabular}

${ }^{\text {a }}$ Data are presented as $\mathrm{n}(\%)$.

'One response missing $(n=25)$.

previous experience with various different types of box simulators. Concerning this judgment, we found that mounting/dismounting, cleaning, transportation, and singlesurgeon training was better than in commercially available endoscopic simulators. On the other hand, most critics were concerned about image light and overall endoscopic environment. These 2 aspects depend largely on webcam's definition and the light source used. In laparoscopy, light source comes from the scope. Thus, shadow is always behind the structures we are focusing. In our homemade simulator, light was coming from all the sides, creating shadows from different directions. As said before, we could use a light incorporated in the webcam, but this would be harder to find and is more expensive. Concerning webcam's definition, our webcam had $640 \times 480$ pixels definition. A higher definition camera could be used, but again it would be more expensive. The majority of participants answered that image light and endoscopic environment provided by our homemade simulator was at least as good as the one provided by commercially available ones.

\section{Conclusions}

We describe 5 easy steps on how to build a homemade low-cost endoscopic simulator. This simple scheme is an affordable way for surgeons and medical students to make their own simulator, comparable with those commercially available, which they should use to practice and perfect their laparoscopic skills.

\section{Authors' Note}

The current study was presented at the May 2011 Annual Meeting of the International Pediatric Endosurgery Group, Prague, Czech Republic.

\section{Declaration of Conflicting Interests}

The author(s) declared the following potential conflicts of interest with respect to the research, authorship, and/or publication of this article: Professor Jorge Correia-Pinto is consultant for Karl Storz, Germany. The remaining authors do not have financial interests to declare. 


\section{Funding}

The author(s) received no financial support for the research, authorship, and/or publication of this article.

\section{References}

1. Spaner SJ, Warnock GL. A brief history of endoscopy, laparoscopy, and laparoscopic surgery. J Laparoendosc Adv Surg Tech A. 1997;7:369-373.

2. Gallagher AG, Smith CD, Bowers SP, et al. Psychomotor skills assessment in practicing surgeons experienced in performing advanced laparoscopic procedures. J Am Coll Surg. 2003;197:479-488.

3. Seymour NE. VR to OR: a review of the evidence that virtual reality simulation improves operating room performance. World J Surg. 2008;32:182-188.
4. Mughal M. A cheap laparoscopic surgery trainer. Ann $R$ Coll Surg Engl. 1992;74:256-257.

5. Gue S. Home-made videoscopic trainer for operative laparoscopic surgery. Aust N Z J Surg. 1995;65:820-821.

6. Chandrasekera SK, Donohue JF, Orley D, et al. Basic laparoscopic surgical training: examination of a low-cost alternative. Eur Urol. 2006;50:1285-1290.

7. Chung SY, Landsittel D, Chon CH, Ng CS, Fuchs GJ. Laparoscopic skills training using a webcam trainer. J Urol. 2005;173:180-183.

8. Beatty JD. How to build an inexpensive laparoscopic webcam-based trainer. BJU Int 2005;96:679-682.

9. Madan AK, Frantzides CT, Shervin N, Tebbit CL. Assessment of individual hand performance in box trainers compared to virtual reality trainers. Am Surg. 2003;69: 1112-1114. 